at the university skin clinic of Heidelberg.] J Dtsch Dermatol Ges 2006;4:308-18. In German.

19 Mahmud E, Brocato M, Palakodeti V, Tsimikas S. Fibromuscular dysplasia of renal arteries: percutaneous revascularization based on hemodynamic assessment with a pressure measurement guidewire. Catheter Cardiovasc Interv 2006;67:434-7.

20 Laforet P, Eymard B. [Exercise-intolerance and exercise-induced rhabdomyolysis: etiology and diagnosis.] Review. Rev Neurol (Paris) 2004;160:217-23. In French.

21 Mora Palma FJ, Ellis HA, Cook DB et al. Osteomalacia in patients with chronic renal failure before dialysis or transplantation. Q J Med 1983;52:332-48.

\title{
Drug-induced disorders of the
}

\section{nervous system}

Mark R Baker MA MB BChir PhD MRCP, Specialist Registrar, Department of Neurology, Royal Victoria Infirmary, Newcastle upon Tyne

Don J Mahad MB ChB PhD MRCP, Specialist Registrar and Wellcome Trust Intermediate Fellow, Mitochondrial Research Group, School of Neurology, Neurobiology and Psychiatry, Medical School, Newcastle upon Tyne

Clin Med 2007;7:170-6

Drug-induced neurological disorders (DINDs) are important because early recognition and drug withdrawal can prevent irreversible damage. This review deals specifically with the recognised neurological complications of prescription-only, single-agent therapy, either at recommended doses or in excess (including overdose and abuse of prescription drugs), and summarises the more important DINDs. Some of the drugs described may also exacerbate neurological disorders that are often subclinical and undiagnosed. Drug interactions can increase the toxicity of single agents; these are well documented elsewhere.

DINDs can occur at initiation, during sudden withdrawal of therapy (eg tramadol withdrawal seizures) and after many months or years of therapy (eg valproate encephalopathy). Treatment is primarily concerned with controlled withdrawal, but toxic levels may necessitate haemodialysis or haemofiltration (eg lithium), specific neutralising therapies (eg Fab fragments in digoxin toxicity) or symptomatic treatments.

Recent detailed and comprehensive reviews of DINDs can be found elsewhere. ${ }^{2,3}$

\section{Seizures}

Seizures may be caused by antipsychotics, antidepressants, antineoplastic agents (alkylators, antimetabolites, vinca alkaloids, ifosfamide and cisplatin), penicillins, cephalosporins, quinolones, antimalarials, aminophylline and allopurinol. They are frequently observed in drug-induced encephalopathy (see below). Other less frequently reported drugs which may induce seizures include

\section{Key Points}

Prescription drugs are an important reversible cause of common neurological disorders

Mitochondrial toxicity is an important mechanism in a number of drug-induced neurological disorders (DINDs)

Early recognition of DINDs and drug withdrawal can prevent irreversible damage

Some DINDs require urgent symptomatic treatment to avoid serious complications

Vitamins and essential trace elements may help to reverse some DINDs

Statin-induced neurological disorders are likely to become more prevalent

Increasing use of novel humanised monoclonal antibodies may cause previously unrecognised DINDs

KEY WORDS: antiretrovirals, drug-induced disorders, mitochondrion, monoclonal antibodies, nervous system, statins 
opioids, baclofen, digoxin, dopaminergic agents, thyroxine and ondansetron. ${ }^{4}$

\section{Drug-induced cerebrovascular disease}

Mechanisms of drug-induced cerebrovascular disease are outlined schematically, with examples, in Fig 1. Antihypertensives and arrhythmogenic agents can cause focal ischaemia, particularly in the elderly, with right shifted autoregulation and underlying atherosclerotic arterial stenoses. ${ }^{2}$ Cancer chemotherapy may cause thromboembolism, haemorrhage, vasopasm or vasculitis; probably because of the synergistic actions of factors secreted by the tumour or products of tumour lysis. In contrast to primary vasculitis, druginduced cerebral vasculitis (Fig 2) involves small arteries and is characterised by polymorphonuclear leucocytic infiltrates without giant cells or granulomata, marked necrosis and marked intimal involvement.

\section{Headache}

Drugs that cause headache usually do so by exacerbating pre-existing migraine, tension headache or chronic daily

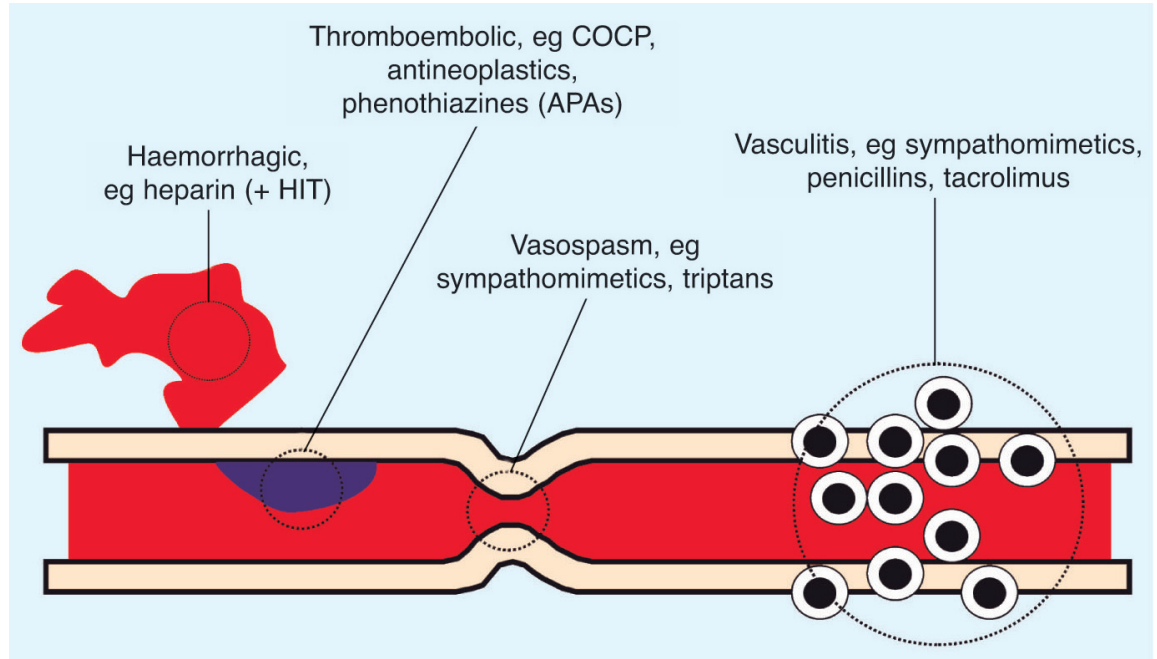

Fig 1. Mechanisms of drug-induced cerebrovascular disease. APA = drug-induced antiphospholipid antibodies; COCP = combined oral contraceptive pill; HIT = heparininduced thrombocytopenia.

headache. Analgesics, including those used to treat headache, may also cause the analgesic overuse syndrome seen most commonly in frequent use of opiate-based analgesia. Drugs used in the treatment of other medical conditions which can cause headache include antidepressants, $\mathrm{H}_{1}$ - and $\mathrm{H}_{2}$-antagonists, antifungals, antihypertensives, betablockers, sulphonamides, intravenous immunoglobulin (IVIg) and oral contraceptive pills (OCPs).
One special cause of headache, usually associated with visual disturbance and papilloedema, is idiopathic intracranial hypertension (IIH). Tetracyclines, retinoids, corticosteroids, oestrogen receptor agonists and antagonists, nonsteroidal anti-inflammatory drugs, growth hormone, cimetidine, nalidixic acid, trimethoprim-sulfamethoxazole, amiodarone and lithium are all associated with IIH.
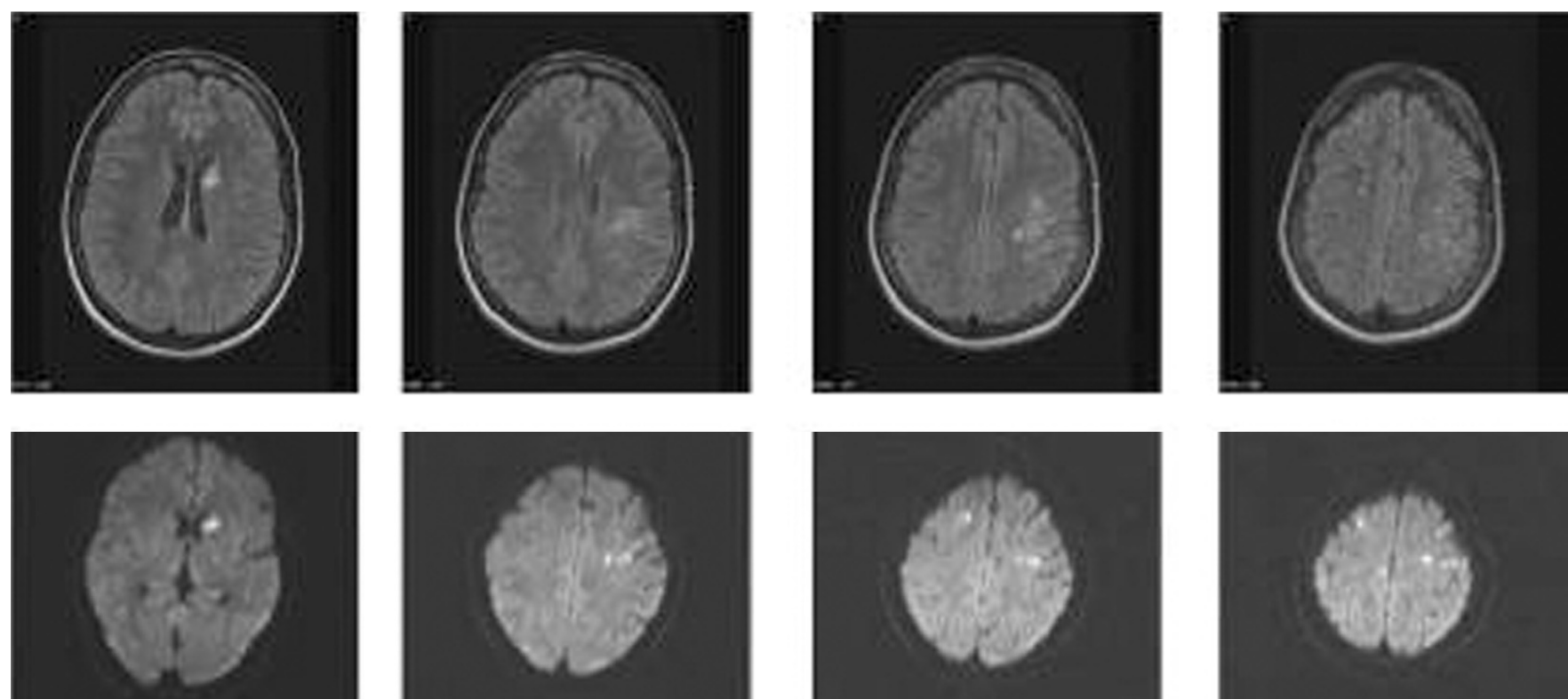

Fig 2. Cocaine-induced cerebrovascular disease. Magnetic resonance image (MRI) brain scans from a patient who presented with strokelike episodes while abusing cocaine: (upper panel) T2-weighted MRI scans; (lower panel) diffusion-weighted images (DWI). DWI demonstrates more widespread ischaemic changes than T2-weighted MRI. 


\section{Encephalopathy}

Drugs that cause encephalopathy and coma do so either by inducing metabolic disturbances (eg hepatic, hypoglycaemic, hyponatraemic or uraemic encephalopathy) or by direct central nervous system (CNS) toxicity (eg anti-epileptic drugs (AEDs), cephalosporins, penicillins, IVIg). Drug-induced coma most commonly occurs at toxic serum concentrations of drug, typically after overdose (eg tricyclic antidepressants (TCAs), opiates, benzodiazepines, AEDs).
Posterior reversible leukoencephalopathy (PRLE) (Fig 3) is associated with seizures and hypertension and commonly occurs in transplant patients treated with ciclosporin or tacrolimus. Other drugs known to cause PRLE include aciclovir, amphotericin B, cisplatin, cytarabine, ifosfamide, and methotrexate.

\section{Dementia}

Anticholinergics, antihypertensives (eg calcium channel blockers, adrenergic agents), AEDs (sodium valproate,

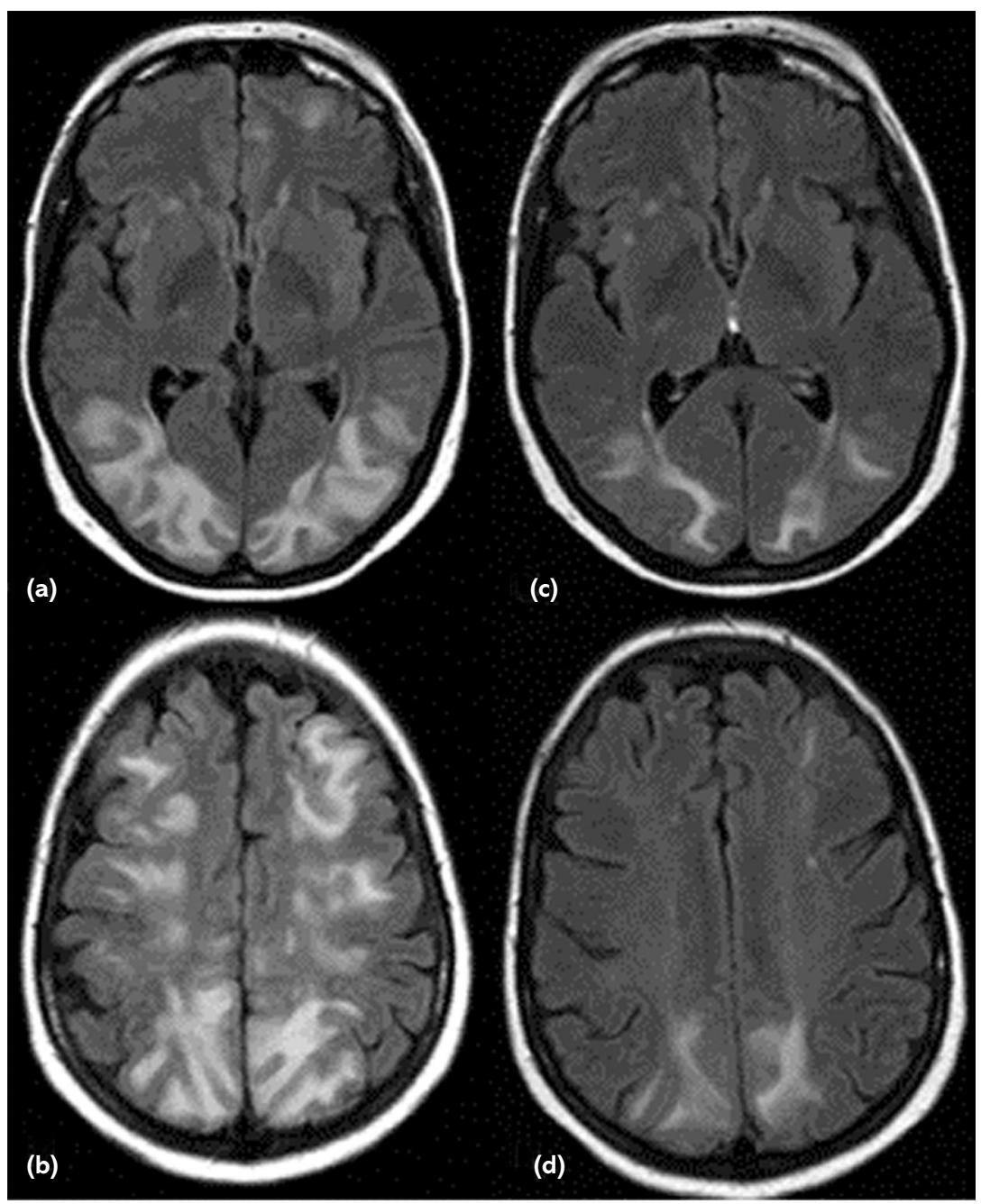

Fig 3. Posterior reversible leukoencephalopathy (PRLE). Fluid attenuated inversion recovery (FLAIR) magnetic resonance image (MRI) sequences obtained from a patient treated with intravenous aciclovir who developed PRLE: (a) and (b) the extent of white matter change during treatment with aciclovir; (c) and (d) similar $\mathrm{MRI}$ sections obtained during the recovery phase after stopping aciclovir. Classically, MRI changes observed in PRLE are limited to the distribution of the posterior circulation and resemble the MRI appearance of hypertensive encephalopathy. PRLE is usually, but not always, associated with hypertension and seizures. phenytoin, lamotrigine), antipsychotics, benzodiazepines, corticosteroids, cytokines (eg interleukins, interferon (IFN)- $\alpha$ ) and desferrioxamine can all cause a reversible condition resembling dementia.

\section{Extrapyramidal disorders}

Drug-induced symmetrical akinetic rigid syndromes are thought to result from monoamine oxidase dysregulation and chronic dopamine depletion after prolonged treatment with phenothiazines, haloperidol, TCAs, metoclopramide, lithium and cinnarizine. Hyperkinetic syndromes may be the result of drug interactions with dopamine transporter proteins. Acute dystonia-dyskinesia (including oculogyric crisis) is commonly caused by antipsychotics, TCAs, AEDs and metoclopramide. Choreo-athetosis is sometimes seen with benzhexol, neuroleptics and OCPs. TCAs and antipsychotics can both cause restless leg syndrome, while dextroamphetamine, methylphenidate and haloperidol can cause motor tics.

Drug withdrawal is the first step but acute symptomatic treatment is also often required (eg iv procyclidine, benztropine or diphenhydramine and iv benzodiazepines in oculogyric crisis). Tardive dyskinesia, which develops after prolonged treatment with phenothiazines, thioxanthines, butyrophenones, dibenzepines, diphenylbutylpiperidines and metoclopramide, can be irreversible. It may be prevented by concomitant treatment with vitamin E, but the evidence is weak. ${ }^{5}$ Patients in whom tardive dyskinesia develops should be converted to alternative antipsychotic medication (eg sulpiride). Symptomatic treatments can also be used (eg tetrabenazine). Patients may complain of akathisia when treated with phenothiazines, butyrophenones, TCAs, L-dopa and monoamine oxidase inhibitors (MAOIs); if severe and persistent, it can be treated with anticholinergics, amantadine or propranolol. ${ }^{6}$

\section{Cerebellar disorders and tremor}

Drug-induced cerebellar syndromes are occasionally irreversible and can be 
caused by AEDs, phenothiazines, MAOIs, thioxanthines, lithium and ciclosporin. Treatment with theophylline, thyroxine, phenothiazines, butyrophenones, amiodarone, adriamycin, cimetidine, terfenadine, lithium, ciclosporin or tacrolimus can sometimes cause postural and kinetic tremor. Resting tremor is occasionally caused by sodium valproate.

\section{Myoclonus}

TCAs, lithium, antipsychotics, dopamine and dopaminergic agonists can all cause myoclonus. Myoclonus persisting after drug withdrawal or reduction can be treated with clonazepam, sodium valproate, piracetam or levetiracetam, but an alternative diagnosis should be considered. ${ }^{7}$

\section{Eye movement disorders}

The most common DIND of eye movements is nystagmus. Horizontal phasic nystagmus can be caused by benzodiazepines, AEDs, MAOIs, salicylates, fenfluramine, TCAs and ototoxic antimicrobials, while downbeat nystagmus is caused by lithium. External ophthalmoplegia (with absent vestibuloocular reflex) is sometimes seen with TCAs, barbiturates, carbamazepine (overdose) and phenytoin. Occasionally, benzodiazepines, fenfluramine, lithium and fluorouracil can cause internuclear ophthalmoplegia.

\section{Cranial neuropathies}

Cytosine arabinoside, ciclosporin and ganciclovir can all induce abducens palsies, but the last two have been described only following allogeneic bone marrow transplantation. Immunological reactions are likely to explain the occurrence of sulfasalazine, insulin-like growth factor-1, didanosine, and HBV vaccine-induced facial palsies. There are several reports of recurrent laryngeal palsy with vincristine chemotherapy, usually in association with peripheral neuropathy; if severe, it can require tracheal intubation. Early withdrawal of therapy is essential to ensure recovery.

\section{Special senses}

\section{Vision}

Blurred vision is a common side effect of medication. Examples of the more common, more serious and occasionally irreversible causes of visual impairment are given in Fig 4(a). Note that agents known to cause PRLE can also cause cortical blindness.

\section{Ototoxicity}

Ototoxic agents are categorised according to vestibular or cochlear toxicity. Putative mechanisms of hair cell toxicity are identified (Fig 4(b)) by abbreviations in parenthesis (mechano-electrical trans- duction (MET), mitochondria (mt) and membrane potential (V)).

\section{Smell and taste}

DINDs of smell and taste can often be corrected by administering zinc and magnesium supplements and phosphodiesterase inhibitors (PDIs) (eg theophylline, caffeine). Both zinc and magnesium are essential to olfactory and gustatory receptor function; many drugs that cause disorders of smell and taste may act by depleting such trace metals. PDIs increase intracellular cyclic adenosine monophosphate levels which are important for intracellular signal transduction in both smell and taste. ${ }^{2}$

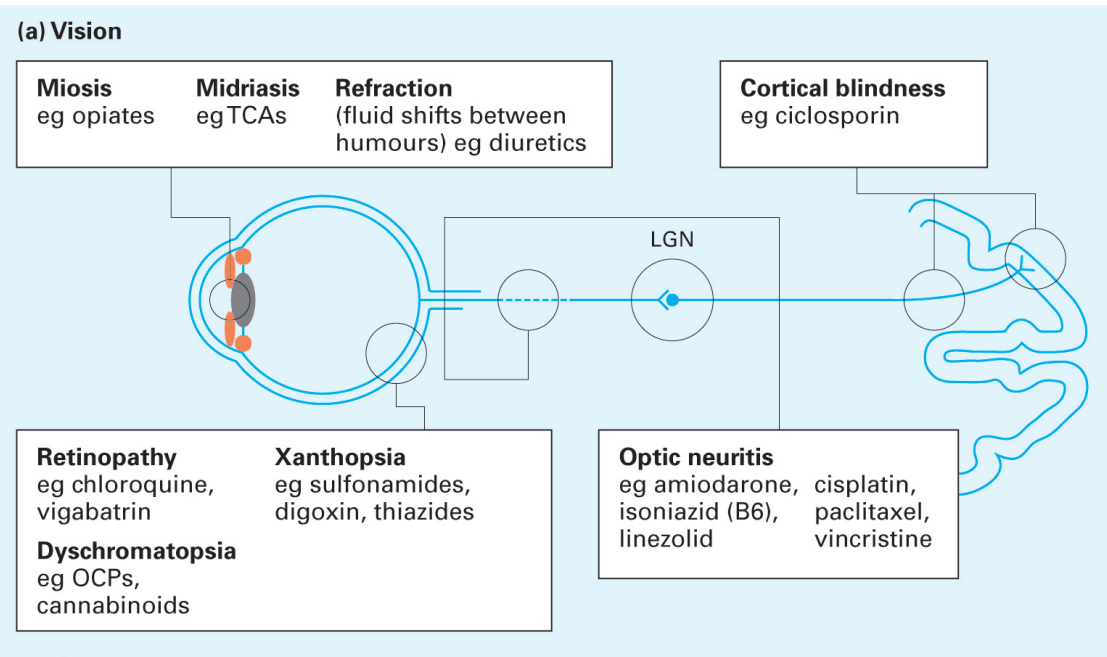

(b) Ototoxicity

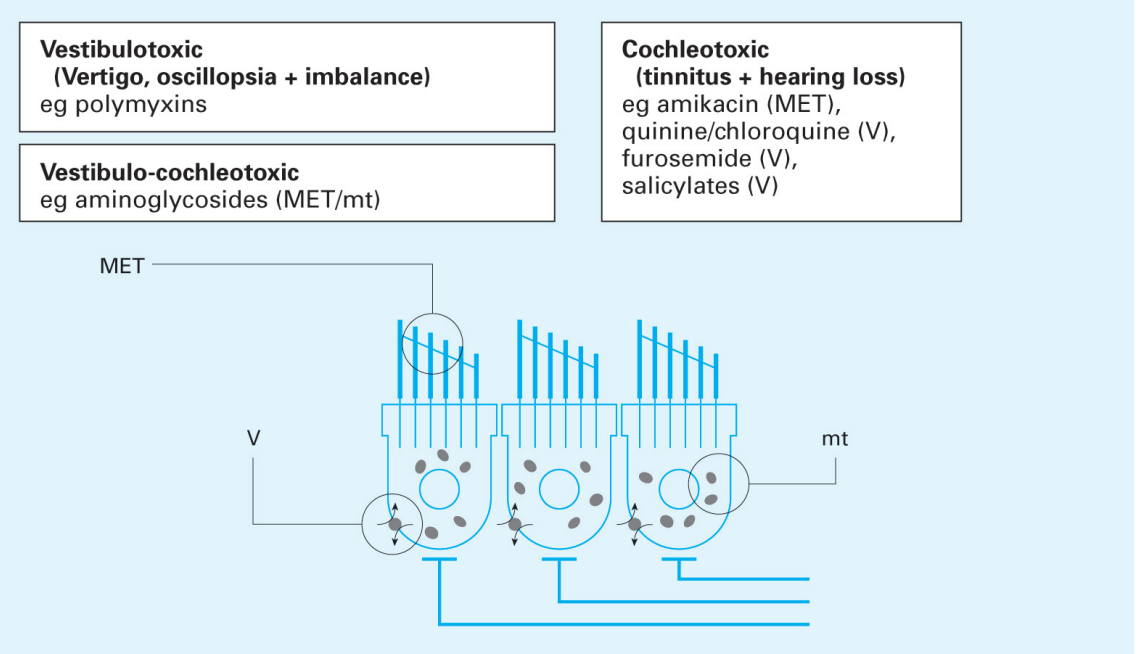

Fig 4. Drug-induced neurological disorders affecting (a) vision and (b) hearing. Possible mechanisms include mechano-electrical transduction (MET), mitochondria (mt) and membrane potential (V). LGN = lateral geniculate nucleus; $\mathrm{OCP}=$ oral contraceptive pill; TCA = tricyclic antidepressant. 


\section{Drug-induced disorders of spinal cord and peripheral nervous system}

Figure 5 provides examples of drugs that can be toxic to the peripheral nervous system and spinal cord, and indicates their sites of toxicity. Drugs that induce subacute myelo-optico-neuropathy are thought to do so by depleting vitamins (particularly B vitamins and vitamin E) or by inhibiting their intracellular cofactors (copper and zinc dependent). Drug-induced neuropathies can be demyelinating (D), axonal (A) or mixed (M) with elements of axonal loss and demyelination. There is little evidence to support specific treatments for druginduced neuropathies (except drug withdrawal), but B vitamins, glutathione and zinc supplements should be considered. ${ }^{2}$

\section{Drug-induced neurological disorders associated with three increasingly commonly used therapies}

Three commonly used therapies associated with DINDs are:

- statins

- antiretroviral agents, and

- humanised monoclonal antibodies (MAb).

\section{Statins}

The frequency of statin-induced neurological disorders is likely to increase in the UK because of their availability offprescription. The major statin-induced neurological disorders are myopathy and small fibre neuropathy.

Mild myopathic symptoms (eg myalgia) have been reported by $5-7 \%$ of patients taking statins. The incidence of severe myotoxicity is $0.44-0.54$ per 10,000 patient-years. ${ }^{7}$ Fortunately, myopathy usually resolves once the statin is withdrawn. Severe myotoxicity typically presents as a necrotising myopathy with proximal weakness and myalgia. In more severe situations there is rhabdomyolysis with the concomitant risks of renal failure.

The incidence of statin-induced neuropathy is approximately 0.3 per 10,000 patient-years. ${ }^{8}$ It can present up to two years after starting treatment. Clinical features include distal lower limb dysaesthesia and anaesthesia, proximal and distal weakness, and fasciculations. In one case series, the neuropathy resolved in $58 \%$ of cases after stopping statin therapy. ${ }^{9}$

Mitochondrial toxicity has been implicated in the pathogenesis of both statininduced myopathy and neuropathy (see below). Statins increase the ratio of lactate to pyruvate, an indirect indication of mitochondrial dysfunction. Cytochrome oxidase-negative fibres and ragged red fibres, hallmarks of mitochondrial myopathy, are seen in muscle biopsy specimens from patients with statin-induced myopathy. ${ }^{10}$ Statins block the synthesis of ubiquinone (coenzyme Q10), an electron-transfer carrier essential to mitochondrial function, which might suggest one putative mechanism.

\section{Antiretrovirals}

The estimated adult HIV prevalence in the UK is approximately $53,000^{11}$ with a significant proportion of HIV-positive individuals treated with highly active antiretroviral therapy (HAART). Nucleoside analogue reverse transcriptase inhibitors (NRTIs) are an essential component of HAART in HIV. NRTIs cause death and dysfunction of highly energy dependent cells in the nervous system and muscle, manifesting as peripheral neuropathy (eg zalcitabine) or myopathy (zidovudine), by inhibiting mitochondrial polymerase gamma and mitochondrial DNA replication (Fig 6). This causes mitochondrial DNA depletion and mutations, oxidative stress and programmed cell death. ${ }^{12}$
Fig 5. Drug-induced disorders of spinal cord and peripheral nervous system. They may be axonal (A), demyelinating (D) or mixed (M). Abs = antibodies; SMON = subacute myelo-optico-neuropathy; TNF = tumour necrosis factor. 
Immune reconstitution inflammatory syndrome (IRIS) is a recognised complication of HAART in HIV. In IRIS there is a paradoxical deterioration of clinical status (eg appearance of progressive multifocal leukoencephalopathy) as the immune system recovers. ${ }^{13}$

\section{Monoclonal antibodies}

MAbs have received considerable media attention in the UK recently, particularly in the context of cancer therapies. Neurological adverse events are well recognised with MABs that target two specific immune molecules:

Tumour necrosis factor. Anti-tumour necrosis factor (TNF) MAbs, adalimumab (hMAb) and infliximab (chimeric antibody against recombinant TNF) are used to treat rheumatoid arthritis, juvenile chronic arthritis, adult psoriatic arthritis, ankylosing spondylitis and Crohn's disease.

Inflammatory demyelinating diseases of the CNS (MS and transverse myelitis) and peripheral nervous system (chronic inflammatory demyelinating polyradiculoneuropathy), aseptic meningitis, bilat- eral anterior toxic optic neuropathy, mononeuritis multiplex, orbital myositis, polymyositis, CNS lupus and vasculitis are recognised events associated with anti-TNF treatment. The inflammatory disorders caused or aggravated by anti-TNF agents may be due to attenuation of organ specific $\mathrm{T}$ cell apoptosis and $\mathrm{T}$ cell signalling. ${ }^{14}$ Multiple sclerosis (MS) patients who respond to IFN- $\alpha$ have increased levels of TNFrelated apoptosis-inducing ligand, a member of the TNF superfamily.

Very late adhesion molecule. Humanised anti-very late adhesion molecule (VLA)-4 MAb (natalizumab) is used in MS and Crohn's disease. Progressive multifocal leukoencephalopathy, an often fatal disease due to infection of CNS glia by Jamestown Canyon virus (JCV), is a rare $(0.1 \%)$ event related to anti-VLA-4 MAb (natalizumab). JC viraemia with natalizumab may be due to effects on bone marrow (release of JCV infected B cells into the circulation) or lack of immune surveillance due to suppression of CD4+ $\mathrm{T}$ cell entry into the CNS. ${ }^{15}$

Other MAbs can cause neurological disorders, but those are less frequent and the mechanisms unclear. For example, OKT3 (muromonab) may cause encephalopathy and seizures but the mechanisms remain speculative.

\section{Mitochondria and drug-induced neurological disorders}

As is apparent from the preceding discussion, the mitochondrion is the site of toxicity common to many DINDs. The schematic diagram in Fig 6 summarises some of the important metabolic loci of susceptibility within the mitochondrion (examples in red text boxes).

Carnitine is necessary for the transport of long chain fatty acids across the inner mitochondrial membrane and is imported into cells by the carnitine transporter, organic cation transporter (OCTN2), located in the plasma membrane. It is then conjugated with fatty-acyl-coenzyme A (CoA) to form fatty-acyl-carnitine by carnitine palmitoyl transferases (CPT) and transferred across inner mitochondrial membrane by carnitine acylcarnitine translocase (CACT). CPT2 then converts fatty-acylcarnitine back to fatty-acyl-CoA for beta
Fig 6. The role of mitochondria in drug-induced neurological disorders.

ADP = adenosine diphosphate; ATP = adenosine triphosphate; CACT = carnitine acylcarnitine translocase;

$\mathrm{Co} A=$ coenzyme $\mathrm{A} ; \mathrm{CoQ}=$ coenzyme $Q ; C P T=$ carnitine palmitoyl transferase; MPT = mitochondrial permeability transition; $\mathrm{NADH}=$ nicotinamide adenine dinucleotide dehydrogenase; $\mathrm{NRTI}=$ nucleoside analogue reverse transcriptase inhibitor; OCTN = organic cation transporter; TCA = tri-carboxylic acid.

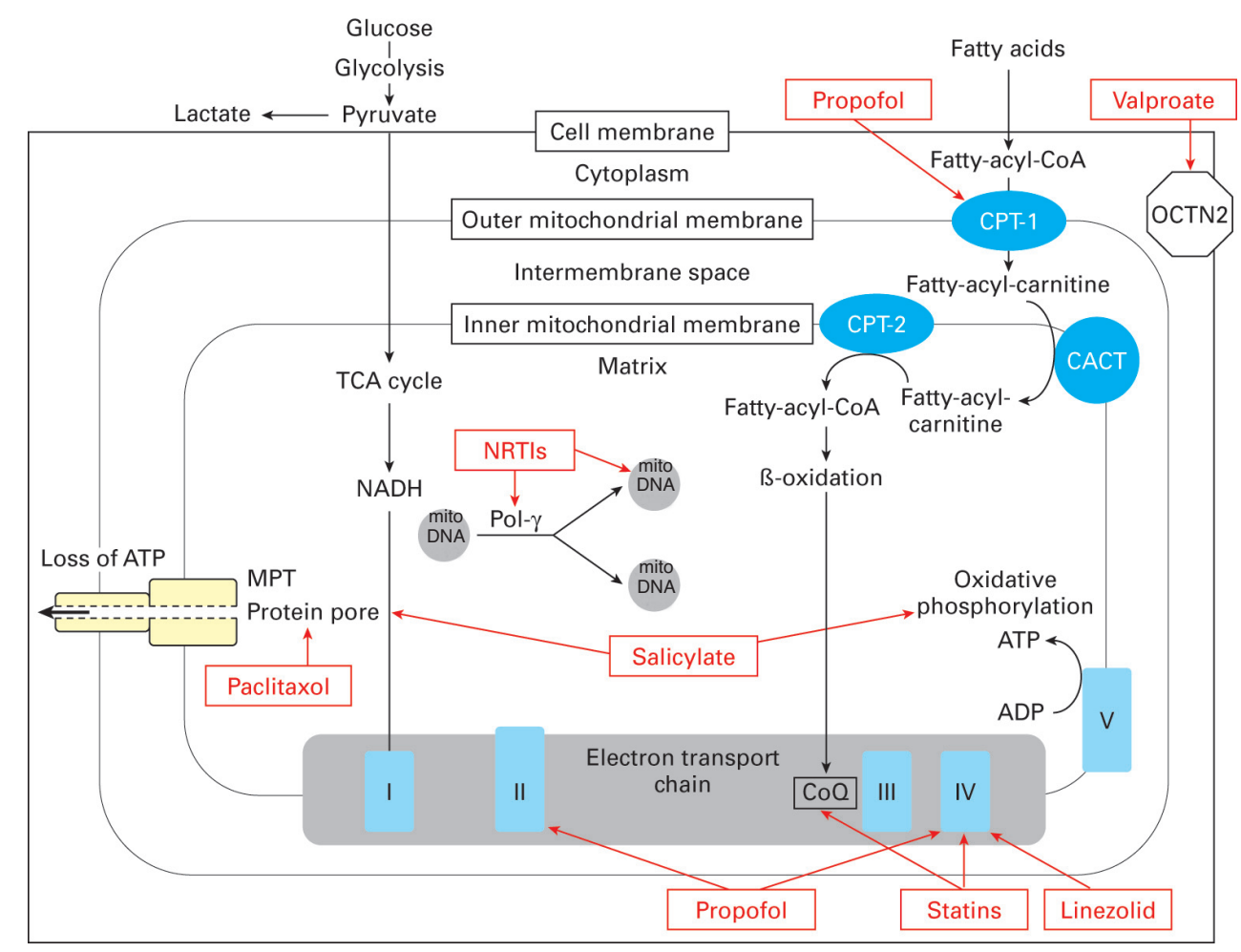


oxidation within the mitochondrion. Primary carnitine deficiency or dysfunction of OCTN2, CPT1, CACT or CPT2 may cause myopathy, rhabdomyolysis, hepatic encephalopathy or hypoketotic, hypoglycaemic coma. Drugs which cause secondary carnitine deficiency can present with a similar clinical picture. Sodium valproate toxicity is associated with carnitine deficiency by inhibiting either carnitine biosynthesis or transport of carnitine into cells, causing encephalopathy with extrapyramidal rigidity, sometimes associated with hyperammonaemia. Valproate encephalopathy can appear years after initiating treatment.

Propofol infusion syndrome due to prolonged exposure to propofol is often fatal and characterised by rhabdomyolysis, bradyarrythmias, renal failure, metabolic acidosis and lipaemic plasma. Propofol inhibits complex II and IV activity as well as CPT1 activity, reducing the entry of fatty-acylcarnitine into mitochondria. ${ }^{16}$

Reye's syndrome, the incidence of which dramatically reduced in the 1980s, presents with acute encephalopathy, raised intracranial pressure and fatty degeneration of the liver; it is associated with the use of aspirin during acute viral illnesses and thought to be caused by salicylate-induced mitochondrial permeability transition. ${ }^{17}$ This process leads to cell death through mitochondrial swelling and uncoupling of oxidative phosphorylation. It is thought to be a consequence of linking of native mitochondrial proteins in outer and inner mitochondrial membranes to form a protein pore, causing loss of adenosine triphosphate production.

Aminoglycoside ototoxicity is associated with a mitochondrial DNA point mutation in the $12 \mathrm{~S}$ ribosome RNA gene (1555A to $G$, which makes rRNA similar to bacterial rRNA), accounting for $15-30 \%$ of aminoglycoside induced deafness. A maternal family history of deafness is an important indicator of susceptibility to aminoglycosides. ${ }^{18}$

Paclitaxol and linezolid are also reported to cause a painful peripheral neuropathy. Paclitaxol is an antiproliferative and pro-apoptotic agent increasingly used to treat cancer. Swollen mitochondria in c-fibres are reported in paclitaxol-associated peripheral neuropathy but the mechanisms are not well known. Linezolid-associated peripheral neuropathy is purportedly due to the inhibition of respiratory chain complex IV activity.

\section{References}

1 Joint Formulary Committee. British national formulary 52. London: BMA and Royal Pharmaceutical Society of Great Britain, 2005.

2 Jain KK. Drug-induced neurological disorders, 2nd edn. Seattle, WA: Hogrefe \& Huber, 2001.

3 Mastaglia FL. Iatrogenic (drug-induced) disorders of the nervous system. In: Aminoff MJ (ed). Neurology and general medicine: the neurological aspects of medical disorders, 2nd edn. Philadelphia: ChurchillLivingstone, 1995:587-614.

4 Garcia PA, Alldredge BK. Drug-induced seizures. Neurol Clin 1994;12:85-99.

5 Soares KVS, McGrath JJ. Vitamin E for neuroleptic-induced tardive dyskinesia. Cachrane Database of Systematic Reviews 2001(4): CD000209.

6 Jimenez-Jimenez FJ, Garcia-Ruiz PJ, Molina JA. Drug-induced movement disorders. Review. Drug Saf 1997;16:180-204.

7 Jimenez-Jimenez FJ, Puertas I, de ToledoHeras M. Drug-induced myoclonus: frequency, mechanisms and management. Review. CNS Drugs 2004;18:93-104.

8 Arora R, Liebo M, Maldonado F. Statininduced myopathy: the two faces of Janus. J Cardiovasc Pharmacol Ther 2006;11: 105-12.

9 Gaist D, Garcia Rodriguez LA, Huerta C, Hallas J, Sindrup SH. Are users of lipidlowering drugs at increased risk of peripheral neuropathy. Eur J Clin Pharmacol 2001;56:931-3.

10 Phan T, McLeod JG, Pollard JD et al. Peripheral neuropathy associated with simvastatin. J Neurol Neurosurg Psychiatry 1995;58:625-8.

11 Phillips PS, Haas RH, Bannykh S et al. Statin-induced myopathy with normal creatine kinase levels. Ann Intern Med 2002;137:581-5.

12 McGarrigle CA, Cliffe S, Copas AJ et al. Estimating adult HIV prevalence in the UK in 2003: the direct method of estimation. Review. Sex Transm Infect 2006;82(Suppl 3): iii78-86.

13 Lewis W, Kohler JJ, Hosseini SH et al. Antiretroviral nucleosides, deoxynucleotide carrier and mitochondrial DNA: evidence supporting the DNA pol gamma hypothesis. AIDS 2006;20:675-84.

14 Battegay M, Nuesch R, Hirschel B,
Kaufmann GR. Immunological recovery and anti-retroviral therapy in HIV-1 infection. Review. Lancet Infect Dis 2006; 6:280-7.

15 Sicotte NL, Voskuhl RR. Onset of multiple sclerosis associated with anti-TNF therapy. Neurology 2001;57:1885-8.

16 Ransohoff RM. Natalizumab and PML. Nat Neurosci 2005;8:1275.

17 Wolf A, Weir P, Segar P, Stone J, Shield J. Impaired fatty acid oxidation in propofol infusion syndrome. Lancet 2001;357:606-7.

18 Lemasters JJ, Nieminen AL, Qian T, Trost LC, Heman B. The mitochondrial permeability transition in toxic, hypoxic and reperfusion injury. Review. Mol Cell Biochem 1997;174:159-65.

19 Fischel-Ghodsian N. Mitochondrial mutations and hearing loss: paradigm for mitochondrial genetics. Review. Am J Hum Genet 1998;62:15-9. 